\title{
Resonator-free tunable sub-MHz spectral dip in the Brillouin gain using spun birefringent fibers
}

Neel Choksi ( $\square$ neel.choksi@mail.utoronto.ca )

University of Toronto https://orcid.org/0000-0002-7583-8815

Yi Liu

University of Toronto

Rojina Ghasemi

University of Toronto

Li Qian

University of Toronto

\section{Research Article}

Keywords: Brillouin scattering, sub-MHz, spun fibers, ultrahigh-Q

Posted Date: April 21st, 2021

DOl: https://doi.org/10.21203/rs.3.rs-440353/v1

License: (9) This work is licensed under a Creative Commons Attribution 4.0 International License.

Read Full License 


\title{
Resonator-free tunable sub-MHz spectral dip in the Brillouin gain using spun birefringent fibers
}

\author{
Neel Choksı ${ }^{1 *}$, YI LIU $^{1}$, Rojina Ghasemi ${ }^{1}$, LI QIAN ${ }^{1^{*}}$ \\ ${ }^{1}$ Department of Electrical and Computer Engineering, University of Toronto, 10 Kings College Road, \\ Toronto, Ontario M5S 3G4, Canada \\ *neel.choksi@mail.utoronto.ca,l.qian@utoronto.ca
}

\begin{abstract}
Ultra-narrow spectral features are desirable for a broad range of applications, and they are conventionally realized using ultrahigh $\mathrm{Q}$ resonant structures. These structures typically require precision fabrication processes, and moreover, since they are passive, they suffer from signal loss. Here, we demonstrate a novel way to achieve sub-MHz tunable spectral dip in the Brillouin gain spectrum of a spun birefringent fiber (SBF) without loss, and without using a resonator. We show that this dip is unique to SBF, where its polarization eigenmodes are elliptical and frequency-dependent, and the dip only occurs when these orthogonal polarization eigenmodes of the SBF (at the respective pump and signal frequencies) are launched in counter-propagating directions. We experimentally demonstrate a $0.72 \mathrm{MHz}$ spectral dip in the Brillouin gain spectrum of a commercial SBF which is to our knowledge, the narrowest SBS spectral feature ever reported. Furthermore, the linewidth, depth, and spectral location of this dip are tunable on demand by controlling the pump frequency, pump power, and the input polarization of the signal. Its simplicity in implementation, its ultra-narrow linewidth, and its tunability can have a wide range of potential applications, from slow-light to microwave photonics.
\end{abstract}

\section{Introduction}

Sub-MHz resonances are highly sought-after for a wide range of applications such as slowlight [1,2] and information storage [3-5], high precision sensing [6], microwave photonics [7-9], spectroscopy [10], frequency stabilization [11], light detection and ranging (LIDAR) [12,13], and optical gyroscopes [14-16]. Demands in these applications have stimulated phenomenal progress in obtaining narrow resonances through platforms like gas-phase atomic systems $[17,18]$, photonic crystal cavities [11,19-21], whispering gallery mode (WGM) [22,23], and microring 
resonators [24-26], slow-light fiber Bragg gratings (FBGs) [2], and phase-shifted FBGs [27]. However, despite significant progress, there remain many challenges with these platforms.

Narrow resonances can be realized in gas-phase atomic systems using electromagnetically induced transparency (EIT) $[17,18]$, but they typically require low temperatures to obtain sub-MHz resonances. Photonic crystal cavities [11, 19-21], WGM [22, 23], and microring resonators [24-26] usually require complex and costly fabrication steps like chemical-mechanical polishing and high temperature anneals to obtain sub-MHz resonances (or equivalently, an ultrahigh Q factor $>10^{8}$ ) [28]. Impedance matched resonator based on $\pi$-phase-shifted FBGs have exhibited narrow resonances [27], but their resonance linewidth is limited to a few MHz due to their intrinsic losses. Recently, efforts have been made to further narrow down the linewidth by using a gain medium to offset the intrinsic loss in a slow-light FBG [2]. But to achieve the narrowest resonance, the FBGs need to be probed with very low signal powers $(<-50 \mathrm{dBm})$, limiting its practical use due to low signal-to-noise ratios. Other than slow-light FBGs, most of these platforms are passive, and their spectral features are usually associated with loss, leading to an undesirable attenuation of the signal.

Stimulated Brillouin scattering (SBS) is another technique to realize narrow spectral features. Typically, the SBS gain linewidth is limited to a few tens of MHz [29,30], and it is challenging to further narrow it down. Recently, efforts have been made to achieve narrower spectral features by realizing an optical analogue of EIT $[3,4]$ and electromagnetically induced absorption (EIA) [31], as well as by combining the SBS gain with high Q resonators [7]. The SBS-based EIT demonstrations $[3,4]$ make use of high $\mathrm{Q}$ resonators to achieve a transparency, and complete transparency is usually difficult to achieve [31]. With EIA [31], it is necessary to precisely control both the amplitudes and the phases of two signals of orthogonal polarizations as well as the amplitude of the pump to achieve exact destructive interference, which is challenging. When SBS is combined with high Q ring resonators [7], it is challenging to precisely align and maintain the alignment of the spectral peak of the Brillouin gain with one of the spectral peaks of the ultrahigh ring resonator. Moreover, the configuration becomes significantly more complex with the added resonator.

In this work, we demonstrate, both theoretically and experimentally, for the first time to our knowledge, a sub-MHz tunable spectral dip in the SBS gain spectrum of a spun birefringent fiber (SBF), at room-temperature, without loss, and without using a resonator. Furthermore, the configuration is extremely simple, no different from a conventional SBS arrangement. An SBF is 
an elliptically birefringent fiber, fabricated by spinning the birefringent preform while drawing the fiber, resulting in two elliptically polarized eigenmodes. The polarization eigenmodes of conventional birefringent fibers (i.e., polarization-maintaining fibers or PMF) are defined by the slow and fast axes of the fiber induced by stress, and therefore they are independent of frequency. In contrast, the elliptical eigenmodes of the SBF are dependent on the twist (spun) period and the beat length of the SBF, and are frequency-dependent. If pump and signal are launched into the SBF in orthogonal polarization eigenmodes, for the pump and signal frequencies, respectively, they remain nearly orthogonal throughout the fiber, and therefore the signal experiences minimal gain. Any minute deviation in the signal frequency causes its eigenmode to deviate from its launch polarization, and due to the positive feedback of the Brillouin gain (polarization pulling effect), the signal polarization is not maintained but rather, pulled toward the pump polarization, causing the signal field to experience a high Brillouin gain [32]. The frequency-dependence of the eigenmode, together with the Brillouin gain, leads to a sub-MHz spectral dip in the Brillouin gain spectrum, which, as we will subsequently demonstrate, is tunable on-demand both in linewidth, depth and frequency.

\section{Theoretical Framework}

An SBF is characterized by its twist rate $\left(k_{t}=\frac{2 \pi}{L_{t}}\right)$ and its unspun linear birefringence $\left(k_{b s / p}=\frac{2 \pi}{L_{b}\left(v_{s / p}\right)}\right)$, where $L_{t}$ and $L_{b}(v)$ are the twist period and the frequency-dependent beat length of the SBF, respectively, $v$ is the frequency of the electric field, and the subscripts $s$ and $p$ denote the signal and pump frequencies, respectively. We neglect the torsion induced birefringence, as SBF is twisted when it is in the molten state with negligible stress due to torsion.

Based on an SBS model for single-mode fiber (SMF) [32-34], we develop the SBS model in SBF with the configuration shown in Fig. 1. The signal field is launched into SBF at $\mathrm{z}=0$, while the pump is launched at $\mathrm{z}=\mathrm{L}$, where $\mathrm{L}$ is the length of SBF. The twist is modeled using a right-handed rotating frame of reference whose coordinates $(\xi, \eta, z)$ are aligned with the fast and slow axes of the fiber locally. Hence, the $(\xi, \eta, z)$ coordinates are rotating with $z$ at the twist rate $k_{t}$. The $(\mathrm{x}, \mathrm{y}, \mathrm{z})$ coordinate system is considered to be the fixed frame of reference, and without the loss of generality, it is assumed that at $z=0$, the fixed frame of reference is aligned with the rotating frame of reference.

In the $(\xi, \eta, z)$ coordinate system, the non-normalized Jones vector for the signal field propagating in $+\mathrm{z}$ direction at a position $\mathrm{z}$ is denoted by $\vec{A}_{r s}(z)$, and for the pump field, 
propagating in -z direction, it is denoted by $\vec{A}_{r p}(z)$. The amplitudes of $\vec{A}_{r}(z)$ are chosen such that their squared values correspond to the optical powers in the mode. In the $(\mathrm{x}, \mathrm{y}, \mathrm{z})$ coordinate system, the non-normalized Jones vectors for the signal and the pump fields are denoted by $\vec{A}_{s}(z)$ and $\vec{A}_{p}(z)$, respectively. The Jones vectors in these two coordinate systems are related by a rotation matrix $\boldsymbol{R}\left(k_{t} z\right)$.

$$
\vec{A}_{r s / p}(z)=\boldsymbol{R}\left(k_{t} z\right) \vec{A}_{s / p}(z)
$$



Fig. 1. The fixed coordinate system $(x, y, z)$ and the right-handed rotating coordinate $\operatorname{system}(\xi, \eta, z)$ are used for modeling of the SBS in spun fiber. The $(\xi, \eta, z)$ coordinates are aligned with the fast and slow axes of the fiber locally, and rotating with $z$ at the twist rate $k_{t}$. At the signal launching end, i.e., $z=0$, the fixed frame of reference is aligned with rotating frame of reference.

The signal frequency $\left(v_{s}\right)$ is downshifted from the pump frequency $\left(v_{p}\right)$ by a frequency $\Omega$ $\left(v_{s}=v_{p}-\Omega\right)$. Using Eq. (1), the coupled differential equations governing the polarization evolution of signal and pump fields in the $(x, y, z)$ coordinate system [32] are converted to the $(\xi, \eta, z)$ coordinate system. In Eqs. (2) and (3), the first term represents the polarization rotation in SBF without gain, and the second term represents the SBS term.

$$
\begin{gathered}
\frac{d \vec{A}_{r s}(z)}{d z}=\frac{d \boldsymbol{T}_{s}(z)}{d z} \boldsymbol{T}_{s}^{\dagger}(z) \vec{A}_{r s}(z)+\gamma\left(\Omega, \Omega_{0}\right)\left(\vec{A}_{r p}\right)\left(\left(\vec{A}_{r p}\right)^{\dagger}(z) \vec{A}_{r s}(z)\right) \\
\frac{d \vec{A}_{r p}(z)}{d z}=\frac{d \boldsymbol{T}_{p}(z)}{d z} \boldsymbol{T}_{p}^{\dagger}(z) \vec{A}_{r p}(z)+\gamma\left(\Omega, \Omega_{0}\right)\left(\vec{A}_{r s}\right)\left(\left(\vec{A}_{r s}\right)^{\dagger}(z) \vec{A}_{r p}(z)\right)
\end{gathered}
$$

Here, $\gamma\left(\Omega, \Omega_{0}\right)[\mathrm{W} \mathrm{m}]^{-1}$ is the Brillouin gain coefficient, which is modelled by a Lorentzian function, where $\Omega_{0}$ is the central Brillouin frequency shift. $\boldsymbol{T}_{s / p}$ is the transfer matrix for the signal (subscript $s$ ) and the pump (subscript $p$ ) field, and it is given by [35-37]:

$$
\boldsymbol{T}_{s / p}(z)=\left[\begin{array}{cc}
\cos \left(k_{b t s / p} z\right)-j \frac{k_{b s / p}}{k_{b t s / p}} \sin \left(k_{b t s / p} z\right) & -\frac{k_{t}}{k_{b t s / p}} \sin \left(k_{b t s / p} z\right) \\
\frac{k_{t}}{k_{b t s / p}} \sin \left(k_{b t s / p} z\right) & \cos \left(k_{b t s / p} z\right)+j \frac{k_{b s / p}}{k_{b t s / p}} \sin \left(k_{b t s / p} z\right)
\end{array}\right],
$$

where $k_{b t s / p}=\sqrt{k_{b s / p}^{2}+k_{t}^{2}}$. The eigenmodes of the transfer matrix $\boldsymbol{T}_{s / p}(z)$ are denoted by $\boldsymbol{S}_{\mathbf{1}}$ 
$\left(\boldsymbol{P}_{\mathbf{1}}\right)$ and $\boldsymbol{S}_{\mathbf{2}}\left(\boldsymbol{P}_{\mathbf{2}}\right)$ at the signal (pump) frequency, which are given by:

$$
\begin{aligned}
& \boldsymbol{S}_{1}: \frac{1}{m_{s}}\left[\begin{array}{c}
i\left(\frac{k_{b s}+k_{b t s}}{k_{t}}\right) \\
1
\end{array}\right], \boldsymbol{S}_{2}: \frac{1}{m_{s}}\left[\begin{array}{c}
i\left(\frac{k_{b s}-k_{b t s}}{k_{t}}\right) \\
1
\end{array}\right] \\
& \boldsymbol{P}_{1}: \frac{1}{m_{p}}\left[\begin{array}{c}
i\left(\frac{k_{b p}+k_{b t p}}{k_{t}}\right) \\
1
\end{array}\right], \boldsymbol{P}_{2}: \frac{1}{m_{p}}\left[i\left(\frac{k_{b p}-k_{b t p}}{k_{t}}\right)\right] \text {, }
\end{aligned}
$$

where $m_{s / p}$ are the normalizing factors.

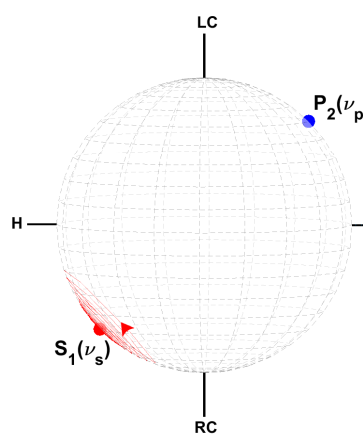

(a)

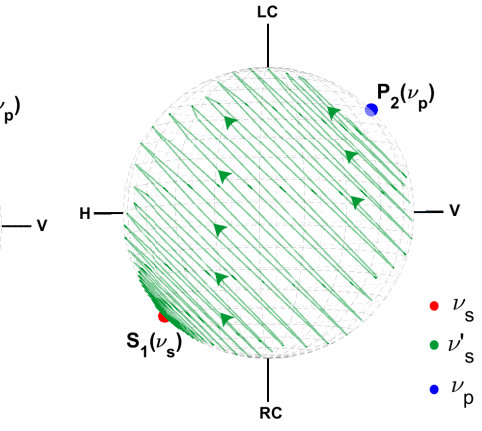

(b)

Fig. 2. Simulated signal polarization evolution (indicated by the red and green traces with arrows on a Poincaré sphere) along the SBF, for different signal frequencies: (a) $v_{s}=v_{p}-\Omega_{o}$ (b) $v_{s}^{\prime}=v_{s}+0.5 \mathrm{MHz}$. For both (a) and (b), the pump launch polarization corresponds to the eigenmode $\mathbf{P}_{2}\left(v_{p}\right)$, and the signal launch polarization corresponds to $\mathbf{S}_{1}\left(v_{s}\right)$, which is an eigenmode for $v_{s}$, but not an eigenmode for the signal frequency of $v_{s}^{\prime}$. Therefore, the signal polarization for case (a) is maintained, and the signal remains nearly orthogonal throughout the fiber. In contrast, the signal polarization for case (b) is not maintained in the SBF, rather, it is pulled towards $\mathbf{P}_{2}\left(v_{p}\right)$. On the Poincaré sphere, $\mathrm{H}$ denotes horizontal polarization, V denotes vertical polarization, LC denotes left-circular polarization, and $\mathrm{RC}$ denotes right-circular polarization. The following parameters are used in the simulation: $v_{p}=192.97 \mathrm{THz}, \Omega_{o}=9.8723 \mathrm{GHz}$, $\mathrm{L}=900 \mathrm{~m}, L_{t}=2.6 \mathrm{~mm}, L_{b}\left(v_{p}\right)=2.6 \mathrm{~mm}$, input pump power $=16.9 \mathrm{dBm}$, and input signal power $=-12 \mathrm{dBm}$.

As can be verified, the eigenmodes $\boldsymbol{S}_{1}$ and $\boldsymbol{P}_{2}$, and $\boldsymbol{P}_{1}$ and $\boldsymbol{S}_{2}$ are nearly orthogonal. (Refer to the blue and red dots on the Poincaré sphere in Fig. 2a). Here we use "nearly" because the 
pump and signal frequencies are different slightly, and therefore the eigenmode for the pump is not exactly orthogonal to the eigenmode for the signal. Nevertheless, these eigenmodes are well maintained throughout their propagation in the SBF [38], even in the presence of Brillouin gain. As a result, the signal and pump are nearly orthogonal throughout the fiber if they are launched into these eigenmodes. (Refer to the red trace on the Poincaré sphere in Fig. 2a for the signal polarization evolution along the fiber). It is important to note here that the signal eigenmode is a function of its frequency. Changing its frequency without changing its launch polarization will result in launching into a non-eigenmode.

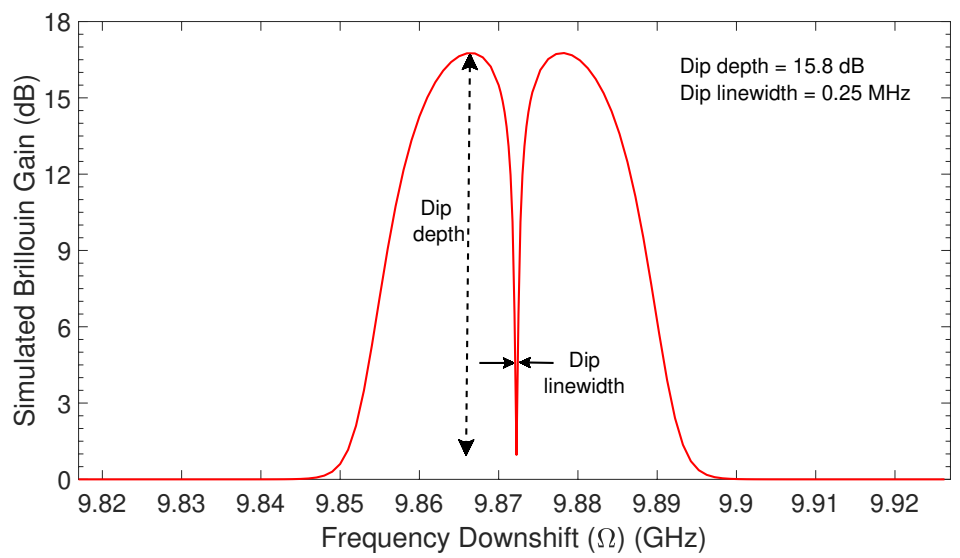

Fig. 3. Stimulated Brillouin gain spectrum of SBF. The input signal polarization is the same for all signal frequencies, and it corresponds to the eigenmode $S_{1}$ at the dip frequency $(9.8723 \mathrm{GHz})$. At any other frequency, the launched signal polarization deviates from the eigenmode for that frequency. The input pump polarization is $\boldsymbol{P}_{\mathbf{2}}$. The linewidth of the dip is $0.25 \mathrm{MHz}$ when measured at $3 \mathrm{~dB}$ from the minimum. The simulation parameters are: $\mathrm{L}=900 \mathrm{~m}, L_{t}=3 \mathrm{~mm}, L_{b}\left(v_{p}\right)=26 \mathrm{~mm}$, input pump power $=16.9 \mathrm{dBm}$, input signal power $=-12 \mathrm{dBm}$, and pump wavelength $=1554.616$ $\mathrm{nm}$.

The signal field experiences minimum Brillouin gain when the signal and pump fields are launched as orthogonal polarization eigenmodes $\left(\mathbf{S}_{1}\left(v_{s}\right)\right.$ and $\left.\mathbf{P}_{2}\left(v_{p}\right)\right)$ of the SBF, and they remain nearly orthogonal along the length of SBF (Fig. 2a). With a minute change in signal frequency from $v_{s}$ to $v_{s}^{\prime}$, the signal launch polarization $\left(\mathbf{S}_{1}\left(v_{s}\right)\right)$ is no longer an eigenmode for $v_{s}^{\prime}$, and the signal polarization will no longer remain orthogonal to the pump polarization due to the polarization pulling effect [32], as shown in Fig. 2b. As a result, the signal at $v_{s}^{\prime}$ experiences a much higher Brillouin gain. Thus this frequency dependence of the eigenmodes along with 
the Brillouin gain leads to a narrow spectral dip in the Brillouin gain spectrum. The simulation result is shown in Fig. 3. A spectral dip with a linewidth of $0.25 \mathrm{MHz}$ (measured at $3 \mathrm{~dB}$ from the minimum) is clearly observed.

It is important to emphasize that this dip is unique to moderately spun birefringent fibers. More specifically, it only occurs when the eigenmodes are elliptical. (Fig. 2 illustrates an example, where $\boldsymbol{S}_{\mathbf{1}}$ and $\boldsymbol{P}_{\mathbf{2}}$ are half way between circular and linear polarization.) As shown in Fig. 5, the dip disappears either when linear birefringence dominates (e.g., in PMF) or when circular birefringence dominates (e.g, when twist rate is very high). In both cases, the eigenmodes become insensitive to frequency. Interestingly, this dip is also not observed in SMF (see Fig. 4), when both beat length and twist period become large. This is why this spectral dip has been elusive, and was never observed in SMF or PMF. This work is the first time such a spectral dip is ever analyzed and observed.

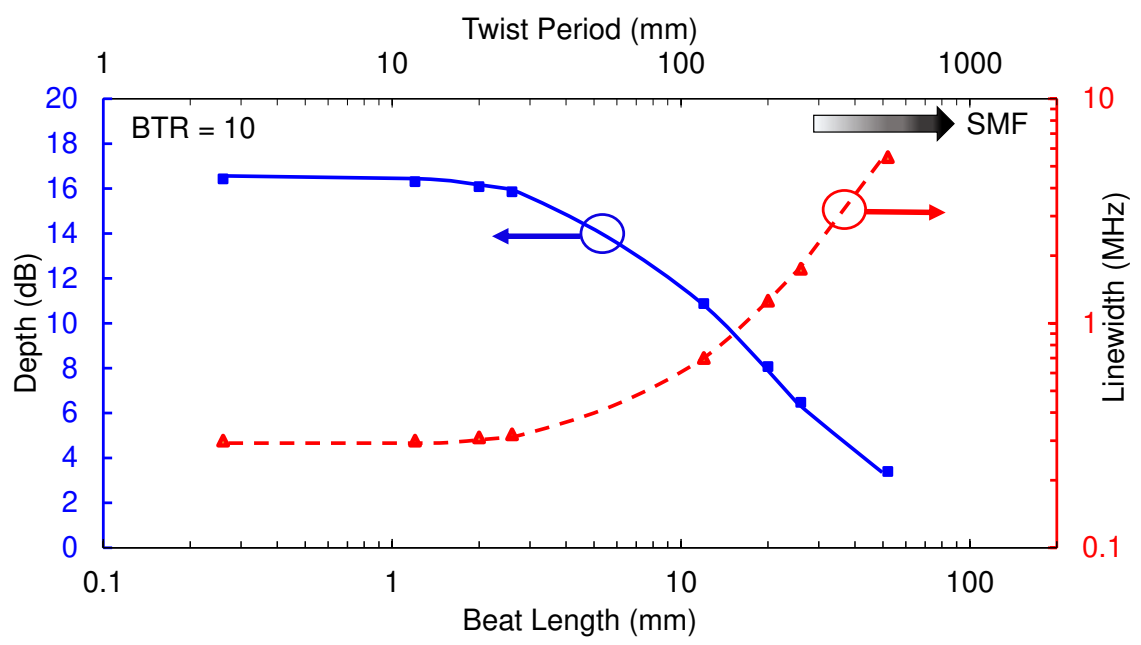

Fig. 4. Simulation result showing the depth (maximum - minimum gain) and the linewidth (measured at $3 \mathrm{~dB}$ from minimum gain) of the spectral dip as a function of $L_{b}\left(v_{p}\right)$ and $L_{t}$ when the birefringence to twist ratio (BTR) is fixed at 10. The simulation parameters are: $\mathrm{L}=900 \mathrm{~m}$, input signal power $=-12 \mathrm{dBm}$, and input pump power $=16.9 \mathrm{dBm}$. The symbols are simulation data points, and the lines are guides to the eye.

To gain more insights into the behaviour of this spectral dip and how it is affected by linear birefringence and twist rate, let us define a Birefringence-to-Twist Ratio (BTR $=k_{b p} / k_{t}=$ $\left.L_{t} / L_{b}\left(v_{p}\right)\right)$. We first analyze the dip depth and linewidth as a function of a fixed BTR of 10 , 
meaning that the unspun linear birefringence is 10 times the twist period. As seen in Fig. 4, for $\mathrm{BTR}=10$, the dip disappears if the beat length (and twist period) becomes too large. In the extreme, the spun fiber becomes an SMF. In the opposite direction, when the beat length (and twist period) decreases, the dip sharpens and deepens, until the dip depth and linewidth converge to limiting values. In other words, for a fixed BTR, when the beat length (and twist period) is sufficiently small, the dip reaches its sharpest limit and no longer narrows or deepens. This is useful for practical considerations. It means that one does not need to fabricate SBFs with impractically high birefringence or twist rate to achieve the narrowest dip. A beat length and twist period of a few $\mathrm{mm}$ is practically achievable.

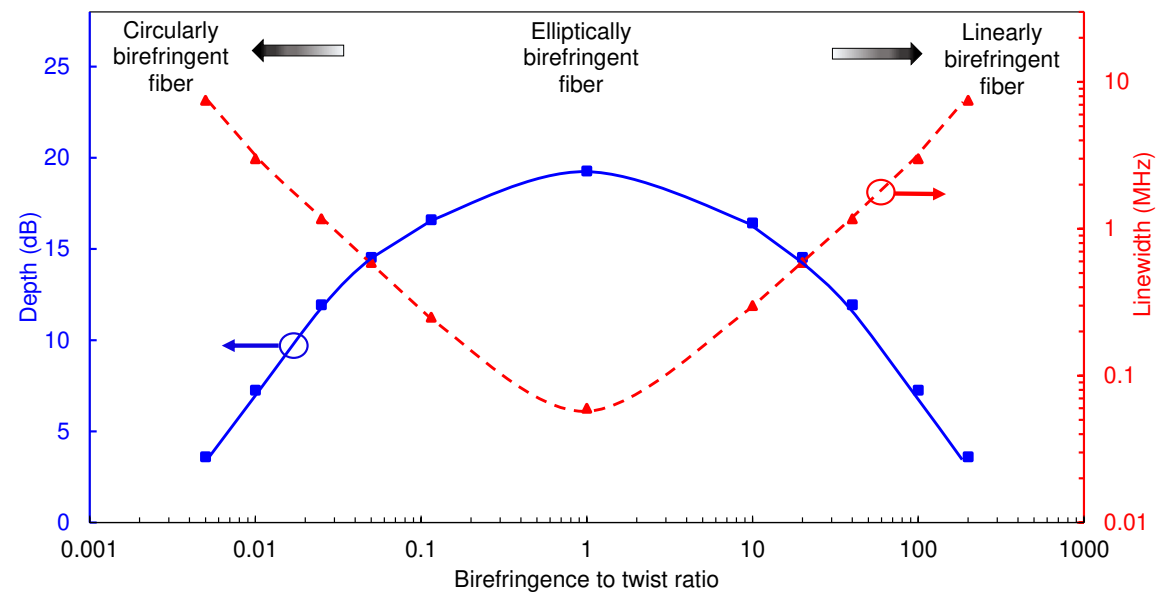

Fig. 5. Simulation result showing the depth (maximum - minimum gain) and the linewidth (measured at $3 \mathrm{~dB}$ from minimum gain) of the narrowest spectral dip realized at a given birefringence to twist ratio (BTR) of SBF. For all the ratios, the beat length of SBF is kept at $0.26 \mathrm{~mm}$. Other parameters used in the simulation are: $\mathrm{L}=900 \mathrm{~m}$, input signal power $=-12 \mathrm{dBm}$, and input pump power $=16.9 \mathrm{dBm}$. The symbols are simulation data points, and the lines are guides to the eye.

Next, we analyze the dip behaviour as a function of BTR (Fig. 5), keeping the beat length sufficiently small $\left(L_{b}=0.26 \mathrm{~mm}\right)$. At very low BTRs (e.g., $<10^{-3}$ ), the SBF behaves like a circularly birefringent fiber, and for high BTRs (e.g., $>10^{3}$ ), the SBF behaves like a linearly birefringent fiber (such as the PMF). At both extremes, the dip disappears. In fact, when the range of the BTR is outside the range of $[0.005,200]$, the dip depth is below $3 \mathrm{~dB}$. The dip linewidth is $<1 \mathrm{MHz}$, and the depth is high $(>10 \mathrm{~dB})$ when BTR lies in the range of [0.05,20]. Predictably, the dip is narrowest (with a linewidth of $0.06 \mathrm{MHz}$ ) and deepest (with a depth of $19 \mathrm{~dB}$ ) at BTR 
$=1$. This dip linewidth is equivalent to a $\mathrm{Q}$-factor greater than 2 billion if a resonator were used.

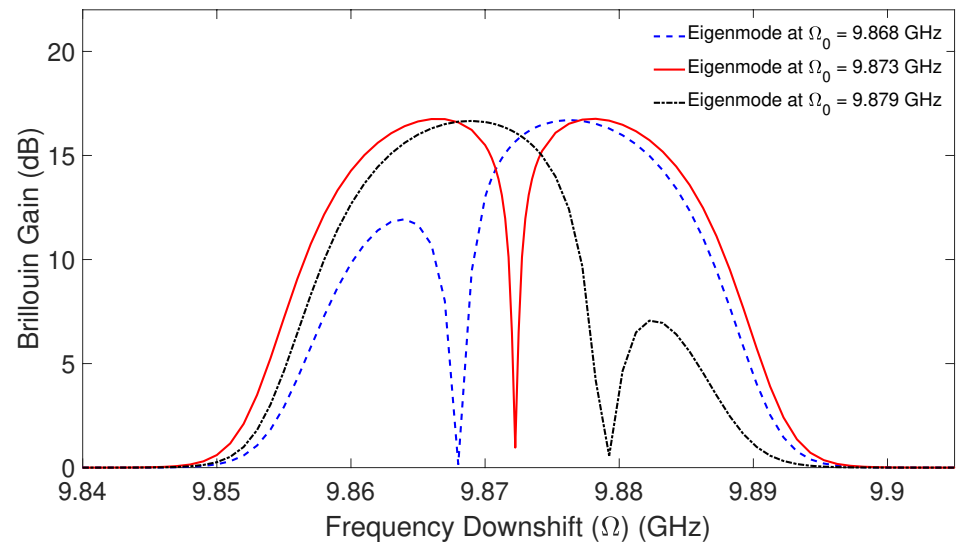

Fig. 6. Simulation result showing the tunability of the spectral position of the dip by varying the input signal polarization, while keeping the pump polarization fixed. Input signal polarizations are the eigenmodes of SBF calculated at different Brillouin frequency shifts $\left(\Omega_{0}\right)$ from the pump. The simulation parameters are: $\mathrm{L}=900 \mathrm{~m}, L_{t}=$ $3 \mathrm{~mm}, L_{b}\left(v_{p}\right)=26 \mathrm{~mm}$, input pump power $=16.9 \mathrm{dBm}$, and input signal power $=-12$ $\mathrm{dBm}$.

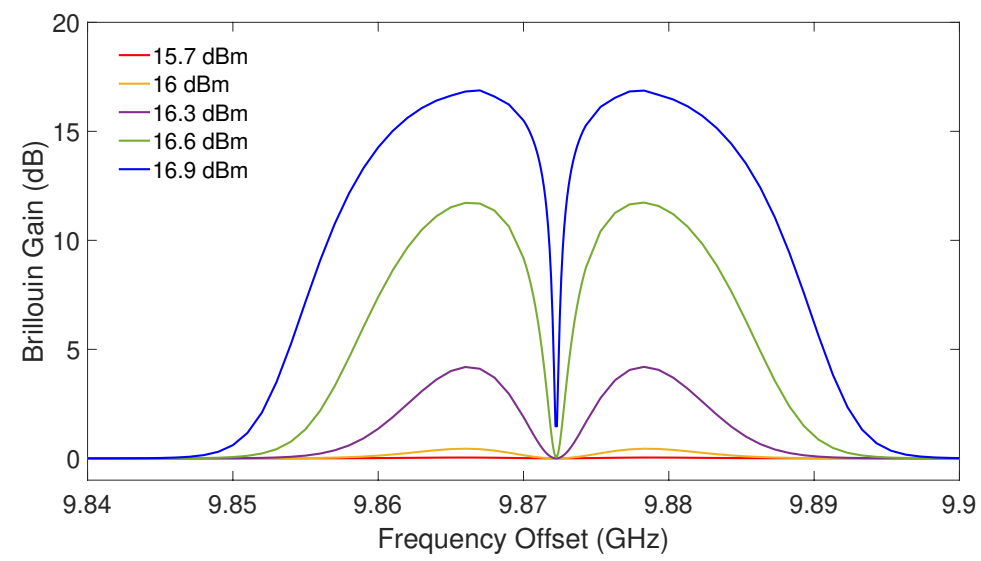

Fig. 7. Simulation result showing the tunability of the spectral linewidth of the dip in the Brillouin gain spectrum of SBF by varying the pump power. Input signal polarizations are the eigenmode of SBF calculated at different downshifted frequencies from the pump. The simulation parameters are: $\mathrm{L}=900 \mathrm{~m}, L_{t}=3 \mathrm{~mm}, L_{b}\left(v_{p}\right)=26 \mathrm{~mm}$, and input signal power $=-12 \mathrm{dBm}$.

The spectral location of this dip can be tuned in real-time (on demand) by changing either 
the pump wavelength or the input polarization, the latter is indicated in Fig. 6. The spectral linewidth of the dip can be tuned by varying the pump power, and it is shown in Fig. 7. With control of the pump frequency, pump power and signal polarization, one has sufficient degrees of freedom to tune the dip frequency, linewidth, and depth, in real-time. This has an important practical significance in microwave photonics.

\section{Experimental Results}

For an experimental demonstration, we use a commercial SBF manufactured by IVG Fiber [39]. The beat length and the twist period of this fiber is $26 \mathrm{~mm}$ and $3 \mathrm{~mm}$, respectively. Note that this fiber does not have the optimal BTR of 1, but it is the only SBF available to us. The experimental arrangement shown in Fig. 8 is used to obtain the Brillouin gain spectrum of SBF. In the first arm, the pump field is amplified and its polarization is adjusted before launching into SBF using a polarization controller. In the second arm, the signal field is amplified, and amplitude-modulated to produce two sidebands. The lower frequency sideband is selected by a tunable filter (aos ultra-narrow filter with $85 \mathrm{~nm}$ bandwidth). The signal polarization is adjusted before launching into $\mathrm{SBF}$, and its frequency is swept over the Brillouin gain range by sweeping the RF driving frequency of the electro-optic modulator (EOM).

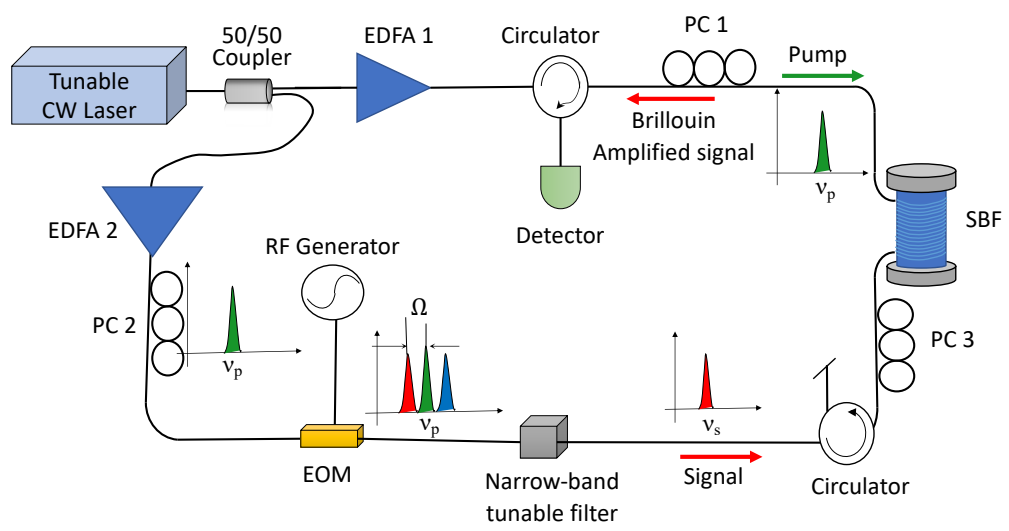

Fig. 8. (a) Experimental set up to measure Brillouin gain: Pump and signal are generated from the same CW laser source. Signal frequency is downshifted from the pump by selecting the lower frequency sideband (red) of the modulated signal after the electro-optic modulator (EOM). Pump and signal polarizations are controlled by the polarization controllers $\mathrm{PC} 1$ and $\mathrm{PC} 3$, respectively. 


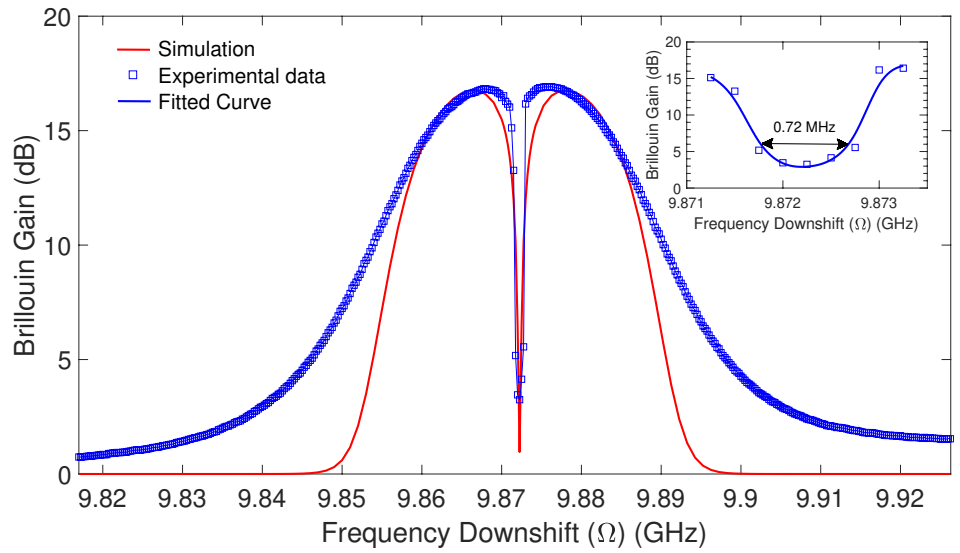

Fig. 9. Comparison of the Brillouin gain spectrum obtained via simulation and experiment. For the simulation, signal and pump fields are launched as orthogonal polarization eigenmodes $\boldsymbol{S}_{\mathbf{1}}$ and $\boldsymbol{P}_{\mathbf{2}}$ into the SBF, for a particular pump frequency $\left(v_{p}\right)$ and the corresponding downshifted signal frequency $\left(v_{s}=v_{p}-\Omega\right)$, in counterpropagating directions. The parameters used for the simulation are similar to the experimental parameters, and they are as following: $\mathrm{L}=900 \mathrm{~m}, L_{t}=3 \mathrm{~mm}, L_{b}\left(v_{p}\right)=$ $26 \mathrm{~mm}$, input pump power $=16.9 \mathrm{dBm}$, and input signal power $=-12 \mathrm{dBm}$.



Fig. 10. Experimental result showing the tunability of the spectral position of the dip in the Brillouin gain spectrum of SBF by varying the input signal polarization, while keeping the pump polarization fixed. The experimental parameters are: $\mathrm{L}=900 \mathrm{~m}, L_{t}$ $=3 \mathrm{~mm}, L_{b}\left(v_{p}\right)=26 \mathrm{~mm}$, input pump power $=16.9 \mathrm{dBm}$, and input signal power $=$ $-12 \mathrm{dBm}$.

The experimental and the simulation results are compared in Fig. 9, and good agreement 
between the two is evident. The simulated dip linewidth is $0.25 \mathrm{MHz}$, whereas the experimental dip linewidth is $0.72 \mathrm{MHz}$, obtained from the least squares polynomial fit of the experimental data near the dip (see inset of Fig. 9). The experimental dip being wider than the simulation dip is likely due to the fact that a lower spectral resolution is used in the measurement $(0.25 \mathrm{MHz})$ than in the simulation $(0.01 \mathrm{MHz})$. Given the experimental dip is actually narrower than the simulation dip in the high gain region, we expect that higher spectral resolution may result in a narrower dip more consistent with the simulation results. The experimental gain shape on the wings of the Brillouin gain deviates from the simulation shape, likely due to the fact that the experimental lineshape is not strictly a Lorentzian lineshape, as is used for the simulation. The tunability of the spectral location of this dip by changing the input polarization is experimentally verified, and the result is presented in Fig. 10.

\section{Conclusion}

In conclusion, we have demonstrated a resonator-free approach of generating a sub-MHz tunable spectral dip at room-temperature and without loss, a unique and never-before-reported SBS spectral dip in spun birefringent fiber. This dip occurs only when the fiber exhibits frequency-dependent polarization eigenmodes, as in a spun birefringent fiber with a moderate birefringence-to-twist ratio. When the pump and signal are launched into the fiber in orthogonal polarization eigenmodes, the signal experiences a minimum gain. A minute deviation of signal frequency results in deviation from the signal polarization eigenmodes, and together with the polarization pulling effect, the signal experiences high Brillouin gain, resulting in a sharp dip feature in the Brillouin gain spectrum. The observed dip linewidth of $0.72 \mathrm{MHz}$ is equivalent to a $\mathrm{Q}$ factor of $\sim 267$ million if a resonator were used. The linewidth, depth and the location of the dip can be tuned on demand by controlling the pump frequency, the pump power, and the input polarization of the signal. Moreover, with an optimal spun birefringent fiber, the dip linewidth can be as low as $0.1 \mathrm{MHz}$, corresponding to a $\mathrm{Q}$ factor greater than 1 billion.

Even though the current analysis of this spectral dip is carried out in fiber, this ultra-narrow feature can potentially be realized in integrated waveguides, using, for instance, chiral birefringent material or a helical waveguide. The gain mechanism is also not limited to SBS. With a broadband polarization-dependent gain (e.g., Raman gain, parametric gain), one might be able to realize a comb of narrow spectral dips. The essential elements required to realize such narrow spectral dips are a rotating birefringence and a polarization-dependent gain. 
The simplicity in the implementation of this technique, as well as the ultra-narrow spectral feature and the easily attainable tunability of the dip, may open up a wide range of potential applications, such as ultrahigh resolution optical sensing, ultra-narrow band tunable optical filters for microwave photonics, slow-light and information storage applications.

\section{References}

1. K. L. Tsakmakidis, O. Hess, R. W. Boyd, and X. Zhang, "Ultraslow waves on the nanoscale," Science 358 (2017).

2. P.-B. Vigneron, T. Boilard, E. Balliu, A. L. Broome, M. Bernier, and M. J. Digonnet, "Loss-compensated slow-light fiber bragg grating with 22-km/s group velocity," Opt. Lett. 45, 3179-3182 (2020).

3. J. Kim, M. C. Kuzyk, K. Han, H. Wang, and G. Bahl, "Non-reciprocal brillouin scattering induced transparency," Nat. Phys. 11, 275-280 (2015).

4. C.-H. Dong, Z. Shen, C.-L. Zou, Y.-L. Zhang, W. Fu, and G.-C. Guo, "Brillouin-scattering-induced transparency and non-reciprocal light storage," Nat. communications 6, 1-6 (2015).

5. C. Wang, X. Jiang, G. Zhao, M. Zhang, C. W. Hsu, B. Peng, A. D. Stone, L. Jiang, and L. Yang, "Electromagnetically induced transparency at a chiral exceptional point," Nat. Phys. 16, 334-340 (2020).

6. A. Arora, M. Esmaeelpour, M. Bernier, and M. J. Digonnet, "High-resolution slow-light fiber bragg grating temperature sensor with phase-sensitive detection," Opt. letters 43, 3337-3340 (2018).

7. H. S. Wen, M. Li, W. Li, and N. H. Zhu, "Ultrahigh-q and tunable single-passband microwave photonic filter based on stimulated brillouin scattering and a fiber ring resonator," Opt. letters 43, 4659-4662 (2018).

8. Y. Liu, A. Choudhary, D. Marpaung, and B. J. Eggleton, "Integrated microwave photonic filters," Adv. Opt. Photonics 12, 485-555 (2020).

9. D. Marpaung, J. Yao, and J. Capmany, "Integrated microwave photonics," Nat. photonics 13, 80-90 (2019).

10. M.-G. Suh, Q.-F. Yang, K. Y. Yang, X. Yi, and K. J. Vahala, "Microresonator soliton dual-comb spectroscopy," Science 354, 600-603 (2016).

11. M. Anderson, N. Pavlov, J. Jost, G. Lihachev, J. Liu, T. Morais, M. Zervas, M. Gorodetsky, and T. Kippenberg, "Highly efficient coupling of crystalline microresonators to integrated photonic waveguides," Opt. letters 43, 2106-2109 (2018).

12. M.-G. Suh and K. J. Vahala, "Soliton microcomb range measurement," Science 359, 884-887 (2018).

13. P. Trocha, M. Karpov, D. Ganin, M. H. Pfeiffer, A. Kordts, S. Wolf, J. Krockenberger, P. Marin-Palomo, C. Weimann, S. Randel et al., "Ultrafast optical ranging using microresonator soliton frequency combs," Science 359, 887-891 (2018).

14. J. Li, M.-G. Suh, and K. Vahala, "Microresonator brillouin gyroscope," Optica 4, 346-348 (2017).

15. W. Liang, V. S. Ilchenko, A. A. Savchenkov, E. Dale, D. Eliyahu, A. B. Matsko, and L. Maleki, "Resonant microphotonic gyroscope," Optica 4, 114-117 (2017)

16. Y.-H. Lai, M.-G. Suh, Y.-K. Lu, B. Shen, Q.-F. Yang, H. Wang, J. Li, S. H. Lee, K. Y. Yang, and K. Vahala, "Earth rotation measured by a chip-scale ring laser gyroscope," Nat. Photonics 14, 345-349 (2020).

17. C. Sayrin, C. Clausen, B. Albrecht, P. Schneeweiss, and A. Rauschenbeutel, "Storage of fiber-guided light in a nanofiber-trapped ensemble of cold atoms," Optica 2, 353-356 (2015).

18. B. Gouraud, D. Maxein, A. Nicolas, O. Morin, and J. Laurat, "Demonstration of a memory for tightly guided light in an optical nanofiber," Phys. review letters 114, 180503 (2015). 
19. A. A. Savchenkov, V. S. Ilchenko, A. B. Matsko, and L. Maleki, "Kilohertz optical resonances in dielectric crystal cavities," Phys. Rev. A 70, 051804 (2004).

20. I. S. Grudinin, V. S. Ilchenko, and L. Maleki, "Ultrahigh optical q factors of crystalline resonators in the linear regime," Phys. Rev. A 74, 063806 (2006).

21. G. Liu, V. S. Ilchenko, T. Su, Y.-C. Ling, S. Feng, K. Shang, Y. Zhang, W. Liang, A. A. Savchenkov, A. B. Matsko et al., "Low-loss prism-waveguide optical coupling for ultrahigh-q low-index monolithic resonators," Optica $\mathbf{5}$, 219-226 (2018).

22. A. Shitikov, I. Bilenko, N. Kondratiev, V. Lobanov, A. Markosyan, and M. Gorodetsky, "Billion q-factor in silicon wgm resonators," Optica 5, 1525-1528 (2018).

23. L. Wu, H. Wang, Q. Yang, Q.-x. Ji, B. Shen, C. Bao, M. Gao, and K. Vahala, "Greater than one billion q factor for on-chip microresonators," Opt. Lett. 45, 5129-5131 (2020).

24. X. Ji, F. A. Barbosa, S. P. Roberts, A. Dutt, J. Cardenas, Y. Okawachi, A. Bryant, A. L. Gaeta, and M. Lipson, "Ultra-low-loss on-chip resonators with sub-milliwatt parametric oscillation threshold,” Optica 4, 619-624 (2017).

25. K. Y. Yang, D. Y. Oh, S. H. Lee, Q.-F. Yang, X. Yi, B. Shen, H. Wang, and K. Vahala, "Bridging ultrahigh-q devices and photonic circuits," Nat. Photonics 12, 297-302 (2018).

26. M. W. Puckett, K. Liu, N. Chauhan, Q. Zhao, N. Jin, H. Cheng, J. Wu, R. O. Behunin, P. T. Rakich, K. D. Nelson et al., "422 million intrinsic quality factor planar integrated all-waveguide resonator with sub-mhz linewidth," Nat. communications 12, 1-8 (2021).

27. M. Jing, B. Yu, J. Hu, H. Hou, G. Zhang, L. Xiao, and S. Jia, "Impedance self-matching ultra-narrow linewidth fiber resonator by use of a tunable $\pi$-phase-shifted fbg," Sci. reports 7, 1-11 (2017).

28. L. Chang, W. Xie, H. Shu, Q.-F. Yang, B. Shen, A. Boes, J. D. Peters, W. Jin, C. Xiang, S. Liu et al., "Ultra-efficient frequency comb generation in algaas-on-insulator microresonators," Nat. communications 11, 1-8 (2020).

29. K. Y. Song, W. Zou, Z. He, and K. Hotate, "All-optical dynamic grating generation based on brillouin scattering in polarization-maintaining fiber," Opt. letters 33, 926-928 (2008).

30. B. J. Eggleton, C. G. Poulton, P. T. Rakich, M. J. Steel, and G. Bahl, "Brillouin integrated photonics," Nat. Photonics 13, 664-677 (2019).

31. A. B. Yelikar, R. Pant et al., "Analogue of electromagnetically induced absorption in the microwave domain using stimulated brillouin scattering," Commun. Phys. 3, 1-11 (2020).

32. A. Zadok, E. Zilka, A. Eyal, L. Thévenaz, and M. Tur, "Vector analysis of stimulated brillouin scattering amplification in standard single-mode fibers," Opt. express 16, 21692-21707 (2008).

33. R. Stolen, "Polarization effects in fiber raman and brillouin lasers," IEEE J. Quantum Electron. 15, 1157-1160 (1979).

34. R. W. Boyd, Nonlinear optics (Academic press, 2019).

35. P. McIntyre and A. W. Snyder, "Light propagation in twisted anisotropic media: Application to photoreceptors," JOSA 68, 149-157 (1978).

36. J.-i. Sakai and T. Kimura, "Birefringence and polarization characteristics of single-mode optical fibers under elastic deformations,” IEEE J. Quantum Electron. 17, 1041-1051 (1981).

37. H. Huang, Microwave Approach to Highly-irregular Fiber Optics (Wiley, 1998).

38. A. Yariv and P. Yeh, Photonics: optical electronics in modern communications (the oxford series in electrical and computer engineering) (Oxford University Press, Inc., 2006).

39. The SBF is drawn from a rotating glass preform, IVG Fiber model LB1300. 


\section{Figures}

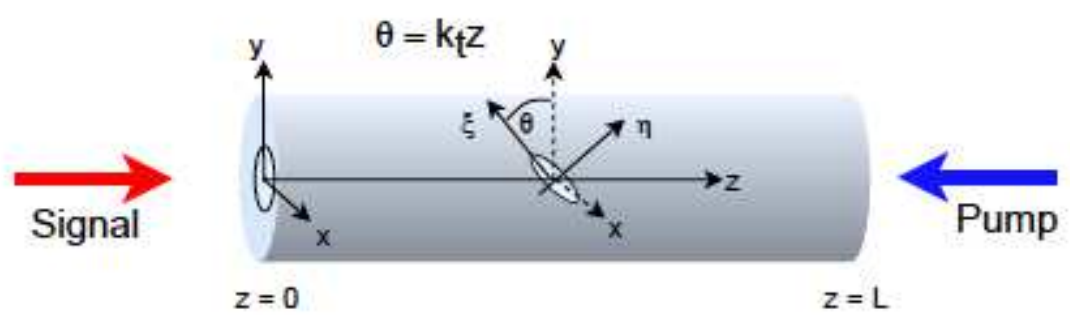

Fig. 1. The fixed coordinate system $(x, y, z)$ and the right-handed rotating coordinate system $(\xi, \eta, z)$ are used for modeling of the SBS in spun fiber. The $(\xi, \eta, z)$ coordinates are aligned with the fast and slow axes of the fiber locally, and rotating with $z$ at the twist rate $k_{t}$. At the signal launching end, i.e., $z=0$, the fixed frame of reference is aligned with rotating frame of reference.

\section{Figure 1}

Please view the figure caption in the figure. 




(a)

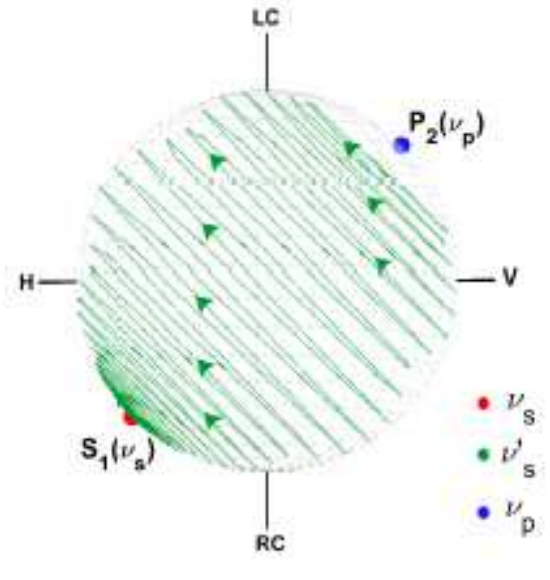

(b)

Fig. 2. Simulated signal polarization evolution (indicated by the red and green traces with arrows on a Poincaré sphere) along the SBF, for different signal frequencies: (a) $v_{s}=v_{p}-\Omega_{o}$ (b) $v_{s}^{\prime}=v_{s}+0.5 \mathrm{MHz}$. For both (a) and (b), the pump launch polarization corresponds to the eigenmode $\mathbf{P}_{2}\left(v_{p}\right)$, and the signal launch polarization corresponds to $\mathrm{S}_{1}\left(v_{s}\right)$, which is an eigenmode for $v_{s}$, but not an eigenmode for the signal frequency of $v_{s}^{\prime}$. Therefore, the signal polarization for case (a) is maintained, and the signal remains nearly orthogonal throughout the fiber. In contrast, the signal polarization for case (b) is not maintained in the SBF, rather, it is pulled towards $\mathbf{P}_{2}\left(v_{p}\right)$. On the Poincaré sphere, $\mathrm{H}$ denotes horizontal polarization, $\mathrm{V}$ denotes vertical polarization, LC denotes left-circular polarization, and RC denotes right-circular polarization. The following parameters are used in the simulation: $v_{p}=192.97 \mathrm{THz}, \Omega_{o}=9.8723 \mathrm{GHz}$, $\mathrm{L}=900 \mathrm{~m}, L_{t}=2.6 \mathrm{~mm}, L_{b}\left(v_{p}\right)=2.6 \mathrm{~mm}$, input pump power $=16.9 \mathrm{dBm}$, and input signal power $=-12 \mathrm{dBm}$.

\section{Figure 2}

Please view the figure caption in the figure. 


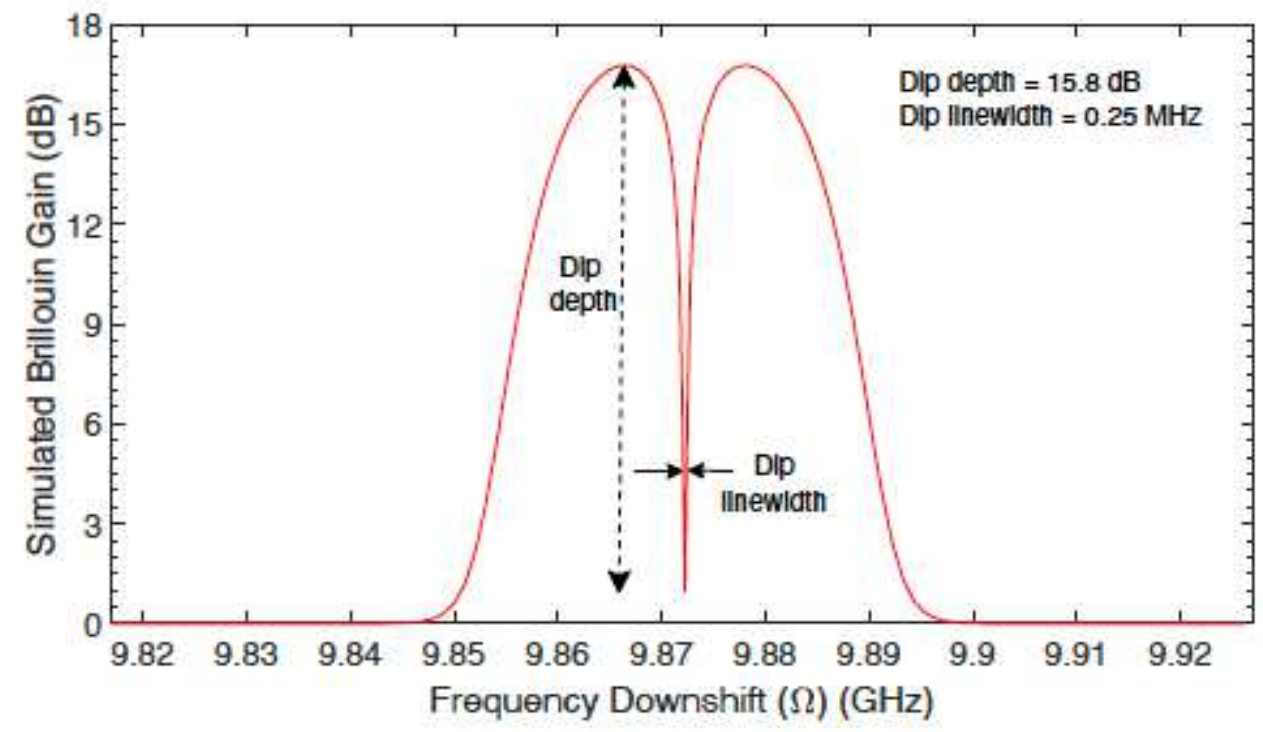

Fig. 3. Stimulated Brillouin gain spectrum of SBF. The input signal polarization is the same for all signal frequencies, and it corresponds to the eigenmode $S_{1}$ at the dip frequency $(9.8723 \mathrm{GHz})$. At any other frequency, the launched signal polarization deviates from the eigenmode for that frequency. The input pump polarization is $\boldsymbol{P}_{\mathbf{2}}$. The linewidth of the dip is $0.25 \mathrm{MHz}$ when measured at $3 \mathrm{~dB}$ from the minimum. The simulation parameters are: $\mathrm{L}=900 \mathrm{~m}, L_{t}=3 \mathrm{~mm}, L_{b}\left(v_{p}\right)=26 \mathrm{~mm}$, input pump power $=16.9 \mathrm{dBm}$, input signal power $=-12 \mathrm{dBm}$, and pump wavelength $=1554.616$ $\mathrm{nm}$.

\section{Figure 3}

Please view the figure caption in the figure. 


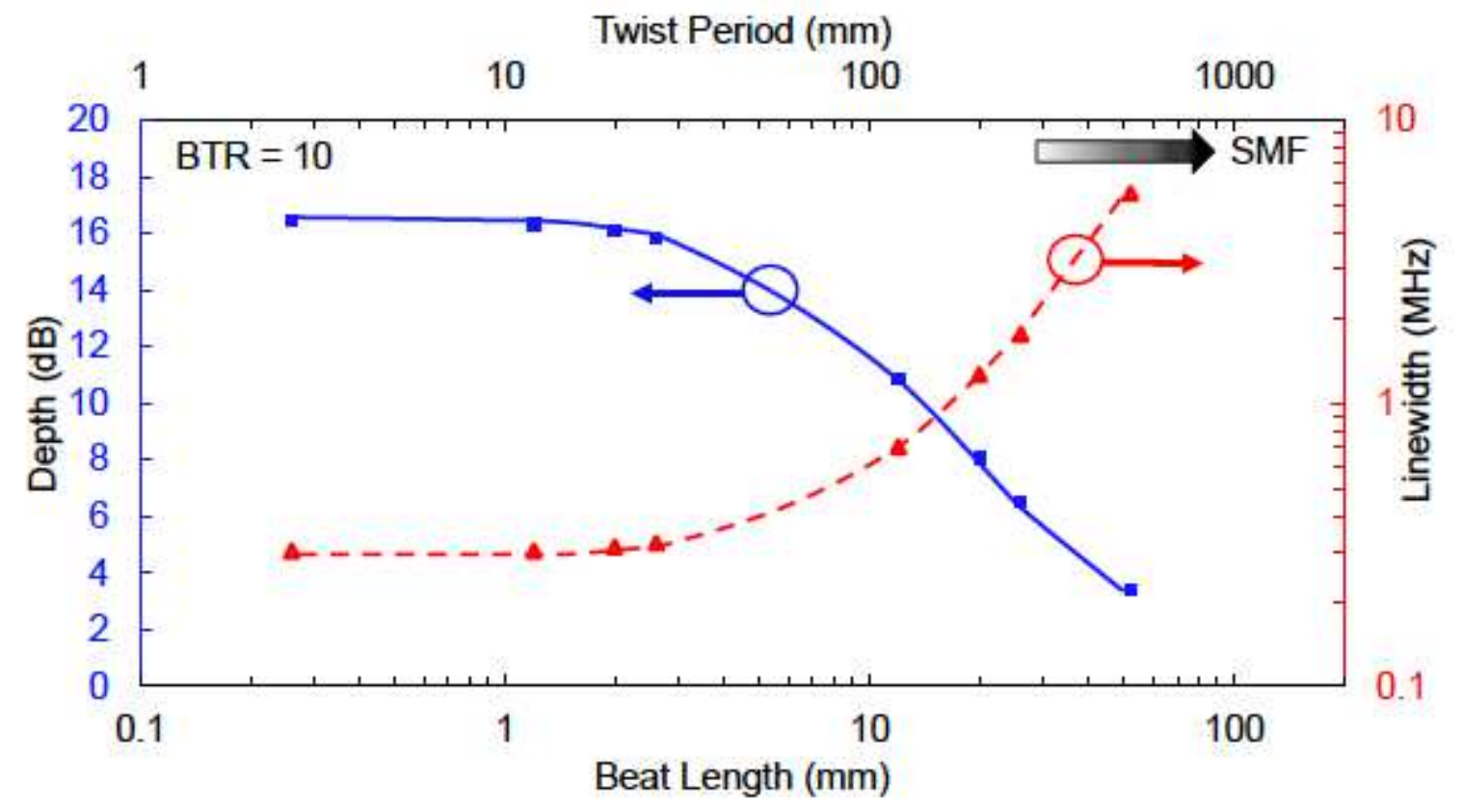

Fig. 4. Simulation result showing the depth (maximum - minimum gain) and the linewidth (measured at $3 \mathrm{~dB}$ from minimum gain) of the spectral dip as a function of $L_{b}\left(v_{p}\right)$ and $L_{t}$ when the birefringence to twist ratio (BTR) is fixed at 10. The simulation parameters are: $\mathrm{L}=900 \mathrm{~m}$, input signal power $=-12 \mathrm{dBm}$, and input pump power $=16.9 \mathrm{dBm}$. The symbols are simulation data points, and the lines are guides to the eye.

\section{Figure 4}

Please view the figure caption in the figure. 




Fig. 5. Simulation result showing the depth (maximum - minimum gain) and the linewidth (measured at $3 \mathrm{~dB}$ from minimum gain) of the narrowest spectral dip realized at a given birefringence to twist ratio (BTR) of SBF. For all the ratios, the beat length of SBF is kept at $0.26 \mathrm{~mm}$. Other parameters used in the simulation are: $\mathrm{L}=900 \mathrm{~m}$, input signal power $=-12 \mathrm{dBm}$, and input pump power $=16.9 \mathrm{dBm}$. The symbols are simulation data points, and the lines are guides to the eye.

\section{Figure 5}

Please view the figure caption in the figure. 




Fig. 6. Simulation result showing the tunability of the spectral position of the dip by varying the input signal polarization, while keeping the pump polarization fixed. Input signal polarizations are the eigenmodes of SBF calculated at different Brillouin frequency shifts $\left(\Omega_{0}\right)$ from the pump. The simulation parameters are: $\mathrm{L}=900 \mathrm{~m}, L_{t}=$ $3 \mathrm{~mm}, L_{b}\left(v_{p}\right)=26 \mathrm{~mm}$, input pump power $=16.9 \mathrm{dBm}$, and input signal power $=-12$ $\mathrm{dBm}$.

\section{Figure 6}

Please view the figure caption in the figure. 


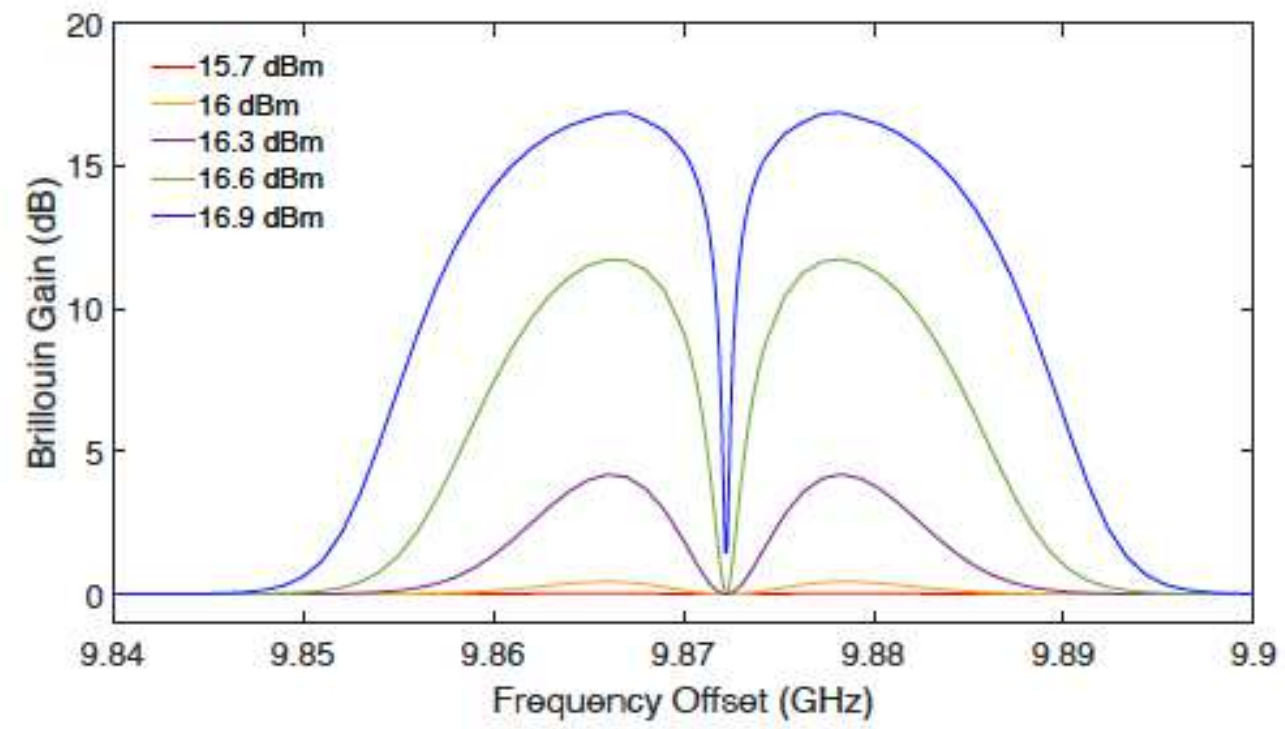

Fig. 7. Simulation result showing the tunability of the spectral linewidth of the dip in the Brillouin gain spectrum of SBF by varying the pump power. Input signal polarizations are the eigenmode of SBF calculated at different downshifted frequencies from the pump. The simulation parameters are: $\mathrm{L}=900 \mathrm{~m}, L_{t}=3 \mathrm{~mm}, L_{b}\left(v_{p}\right)=26 \mathrm{~mm}$, and input signal power $=-12 \mathrm{dBm}$.

\section{Figure 7}

Please view the figure caption in the figure. 


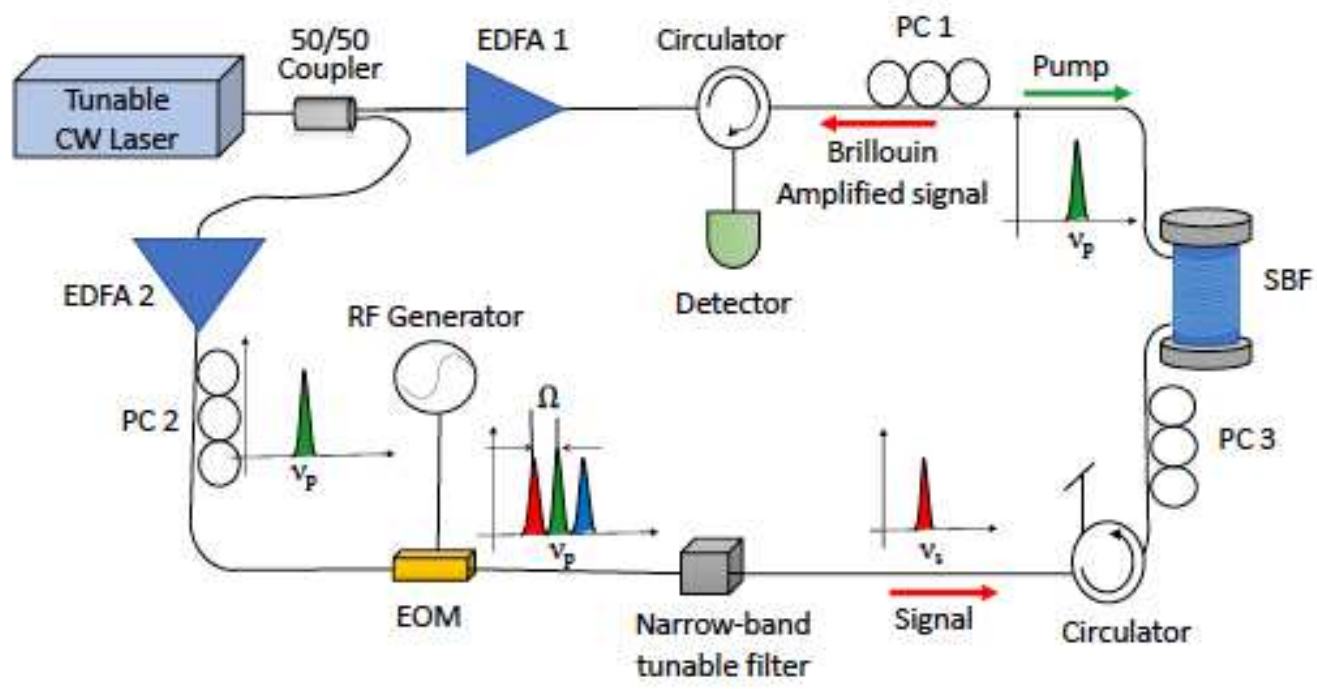

Fig. 8. (a) Experimental set up to measure Brillouin gain: Pump and signal are generated from the same CW laser source. Signal frequency is downshifted from the pump by selecting the lower frequency sideband (red) of the modulated signal after the electro-optic modulator (EOM). Pump and signal polarizations are controlled by the polarization controllers $\mathrm{PC} 1$ and $\mathrm{PC} 3$, respectively.

\section{Figure 8}

Please view the figure caption in the figure. 


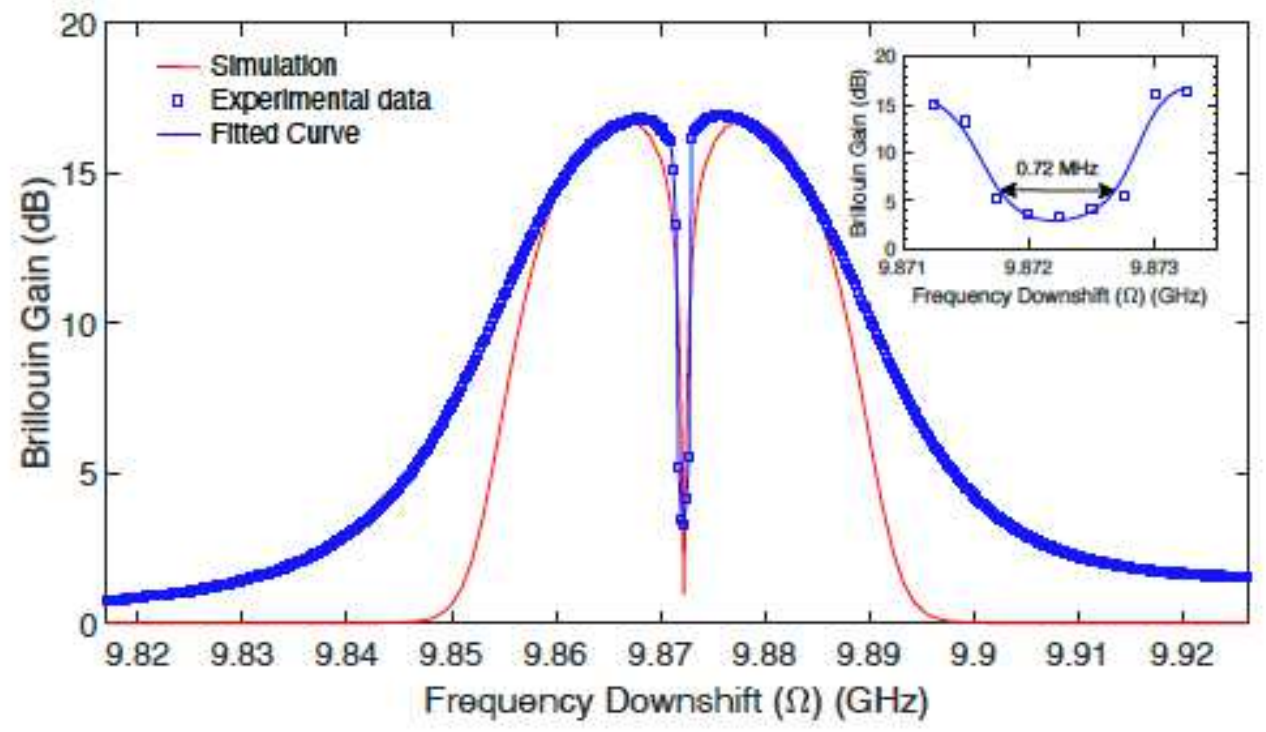

Fig. 9. Comparison of the Brillouin gain spectrum obtained via simulation and experiment. For the simulation, signal and pump fields are launched as orthogonal polarization eigenmodes $S_{1}$ and $\boldsymbol{P}_{2}$ into the SBF, for a particular pump frequency $\left(v_{p}\right)$ and the corresponding downshifted signal frequency $\left(v_{s}=v_{p}-\Omega\right)$, in counterpropagating directions. The parameters used for the simulation are similar to the experimental parameters, and they are as following: $\mathrm{L}=900 \mathrm{~m}, L_{t}=3 \mathrm{~mm}, L_{b}\left(v_{p}\right)=$ $26 \mathrm{~mm}$, input pump power $=16.9 \mathrm{dBm}$, and input signal power $=-12 \mathrm{dBm}$.

\section{Figure 9}

Please view the figure caption in the figure. 


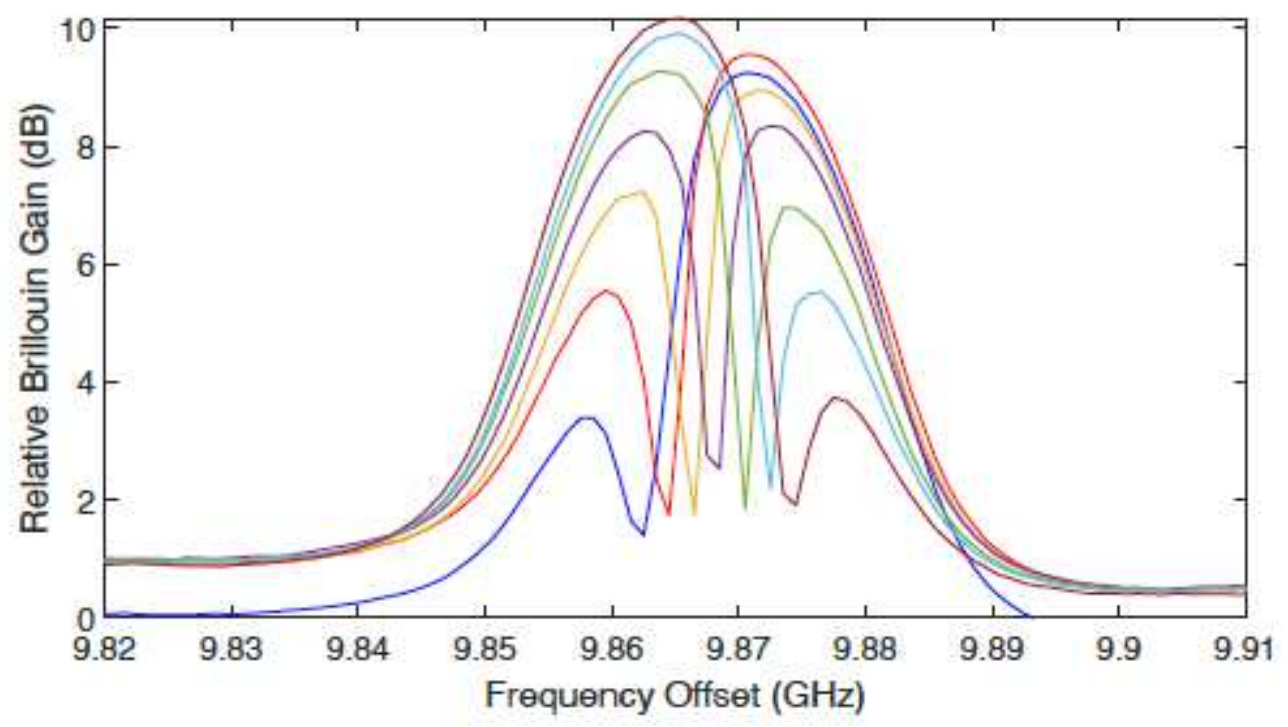

Fig. 10. Experimental result showing the tunability of the spectral position of the dip in the Brillouin gain spectrum of SBF by varying the input signal polarization, while keeping the pump polarization fixed. The experimental parameters are: $\mathrm{L}=900 \mathrm{~m}, L_{t}$ $=3 \mathrm{~mm}, L_{b}\left(v_{p}\right)=26 \mathrm{~mm}$, input pump power $=16.9 \mathrm{dBm}$, and input signal power $=$ $-12 \mathrm{dBm}$.

\section{Figure 10}

Please view the figure caption in the figure. 